\title{
Value of diffusion weighted imaging in the differential diagnosis of benign and malignant breast lesions at 3.0T MRI
}

\author{
X.Y. Jiang, Q.Z. Xie, X.S. Cao, T. Gao, C.Y. Xing and X.L. Li \\ Department of Radiology, Affiliated Hospital of Binzhou Medical College, \\ Bingzhou, Shandong, China \\ Corresponding author: X.-Y. Jiang \\ Email: jiangxing_yue@126.com
}

Genet. Mol. Res. 13 (3): 7773-7779 (2014)

Received July 24, 2013

Accepted December 13, 2013

Published September 26, 2014

DOI http://dx.doi.org/10.4238/2014.September.26.15

\begin{abstract}
The aim of this study was to investigate the correlation between apparent diffusion coefficients (ADCs), the relative apparent diffusion coefficient (rADC), and the pathological prognostic factor human epidermal growth factor receptor 2 (HER-2) in patients with breast cancer. A total of 64 women with breast cancer underwent breast diffusion-weighted imaging. HER-2 expression was detected in histological specimens. The ADC value, rADC value, and HER2 level were determined. The ADC and $\mathrm{AADC}$ values of the breast cancer group were $1.1495 \pm 0.1499 \times 10^{-3}$ and $0.6602 \pm 0.0853$, respectively. The differences in the ADC and $\mathrm{rADC}$ values between the two groups were statistically significant. There was no correlation between the ADC value and HER-2 expression in patients with breast cancer $(r=-0.508, P=0.043)$. However, the $\mathrm{rADC}$ value eliminated
\end{abstract}


the individual differences to some extent. Therefore, compared to the ADC value, the rADC value had a better correlation with HER-2 expression.

Key words: Breast cancer; Magnetic resonance imaging; Genes; HER-2

\section{INTRODUCTION}

In recent years, breast cancer has become a common clinical disease. The traditional breast cancer diagnostic techniques include mammography and ultrasonography. The interest in functional magnetic resonance imaging (MRI) for breast cancer diagnosis has increased because of its high soft tissue resolution, and functional MRI has become widely used in clinical practice. In addition, diffusion-weighted imaging (DWI) has also attracted increasing attention. This technique illustrates the movement of water molecules in tissue due to random thermal motion, i.e., Brownian motion, and it is a functional imaging modality. The apparent diffusion coefficient $(\mathrm{ADC})$ is a very useful parameter in the diagnosis of breast lesions by DWI. However, the relative apparent diffusion coefficient (rADC) is rarely used in the diagnosis of breast disease. Human epidermal growth factor receptor 2 (HER-2) expression is a significant prognostic factor for breast cancer. Thus, this study assessed the correlations between the ADC value, rADC value, and HER-2 expression.

\section{MATERIAL AND METHODS}

\section{General data}

This study included clinically palpable breast cancers treated in our hospital between June 2009 and April 2011. In total, 64 patients underwent MRI for 64 lesions. All patients were women, ranging in age from 28 to 55 years (mean age, 36.5 years). All patients had confirmed diagnoses of breast cancer based on the pathological examination of surgical specimens. There were 60 cases of invasive ductal carcinomas, 3 cases of poorly differentiated adenocarcinomas, and 1 case of medullary cephaloma. A comparative group of 60 normal women ranging in age from 24 to 50 years (mean age, 30.7 years) was also recruited.

\section{Inspection method}

All MR imaging examinations were performed with a 1.5-T imaging unit (Signa Excite II; GE Healthcare, Waukesha, WI, USA) using a dedicated eight-channel bilateral breast coil in the axial orientation. Fast spin-echo T1-weighted imaging [repetition time (TR) 480 $\mathrm{ms}$, echo time (TE) $10 \mathrm{~ms}$ ] and T1-weighted imaging with fat suppression (TR $3200 \mathrm{~ms}$, TE $85 \mathrm{~ms}$ ) were conducted with a slice thickness of $5 \mathrm{~mm}$, a 1-mm barrier, a matrix size of 256 $\mathrm{x} 160$. The DWI imaging was performed with an echo-planar imaging sequence with the following parameters: TR, $5000 \mathrm{~ms}$; TE, $63.2 \mathrm{~ms}$; slice thickness, $4 \mathrm{~mm}$; interval, $2 \mathrm{~mm}$, matrix, $128 \times 128$. The field-of-view was $28 \mathrm{~cm}$ x $28 \mathrm{~cm}$, and the Diffusion gradients were applied in six directions with $\mathrm{b}=0$ and $800 \mathrm{~s} / \mathrm{mm}^{2}$. 


\section{Image analysis}

Using the Functool software of the MRI scanner (Signa Excite II; GE Healthcare), the optimal layer of the lesion was selected, and the diffusion coefficients were measured for $a \mathrm{~b}$ value of $800 \mathrm{~s} / \mathrm{mm}^{2}$ with a region of interest (ROI) of $15-20 \mathrm{~mm}^{2}$. The ADC of a normal gland in the relevant mirror image of the opposite side of the breast was measured, and the ratios of the two values were used as the rADC. The same ROI was used for the control group for rADC measurements.

\section{HER-2 inspection}

HER-2 expression in the surgical pathology specimen was measured by immunohistochemistry with SP method according to the manufacturer protocol. The assessment of the result of HER-2 expression followed the standards of the Hercept Test and the latest clinical laboratory standard in Europe and the US (Ellis et al., 2000; Cell Markers and Cytogenetics Committees, College of American Pathologists, 2002). A positive result was identified by expression in the cell membrane as follows: negative $(-)=$ no staining; weakly positive $(+)=$ less than $10 \%$ of the area displays positive and discontinuous membrane staining; moderately positive $(++)=$ more than $10 \%$ of the area displays positive and discontinuous membrane staining; and strongly positive $(+++)=$ more than $10 \%$ of the area exhibits positive and continuous membrane staining.

\section{Statistical analysis}

All data were statistically analyzed using the SPSS16.0 software. Differences between the ADC values and $\mathrm{rADC}$ values in the breast cancer and control groups were assessed using the Student $t$-test at a significance level of $\alpha=0.05$. Spearman correlation analysis was adopted to analyze the correlations between the ADC value, rADC value, and HER-2 expression.

\section{RESULTS}

A two independent samples t-test identified significant differences in ADC and rADC values between the two groups $(t=4.23, \mathrm{P}<0.001$; Table 1$)$.

Table 1. Comparison of the diffusion-weighted imaging results between the breast cancer and control groups.
\begin{tabular}{lccc}
\hline Group & No. of cases & ADC $\left(\times 10^{-3} \mathrm{~mm}^{2} / \mathrm{s}\right)$ & rADC \\
\hline Breast cancer & 64 & $1.1495 \pm 0.1499$ & $0.6602 \pm 0.0853$ \\
Control group & 60 & $1.7658 \pm 0.1520$ & $1.0782 \pm 0.1278$ \\
\hline
\end{tabular}

$\mathrm{ADC}=$ apparent diffusion coefficient; $\mathrm{rADC}=$ relative apparent diffusion coefficient.

Although the ADC value was not correlated with HER-2 expression in the breast cancer group $(\mathrm{P}=0.923)$, a correlation between the rADC value and HER-2 expression was noted $(\mathrm{r}=-0.508, \mathrm{P}=0.043$; Figures 1-2, Table 2). 


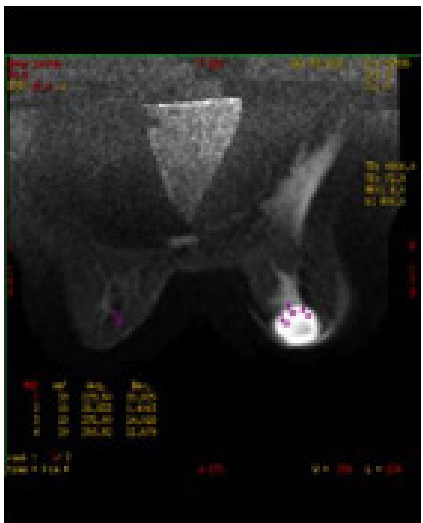

A

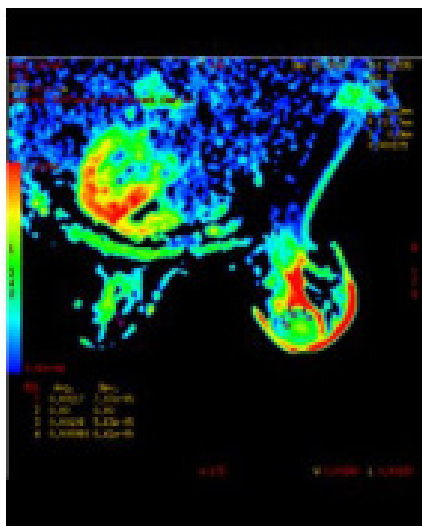

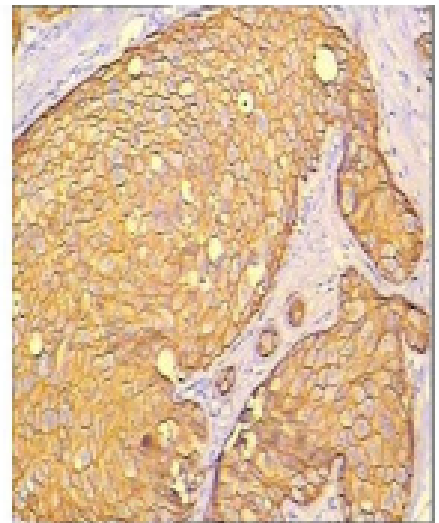

C

Figure 1. Female patient, 58 years, invasive ductal carcinoma. A. Diffusion-weighted imaging shows an obvious high signal in the lesion of the right breast. B. Corresponding apparent diffusion coefficient (ADC) map: the $\mathrm{ADC}$ value is $1.081 \times 10^{-3} \mathrm{~mm}^{2} / \mathrm{s}$, the region of interest area is approximately $19 \mathrm{~mm}^{2}$, and the relative apparent diffusion coefficient is 0.670 . C. Strongly positive immunohistochemical staining for human epidermal growth factor receptor-2 (+++) (SP 400X).

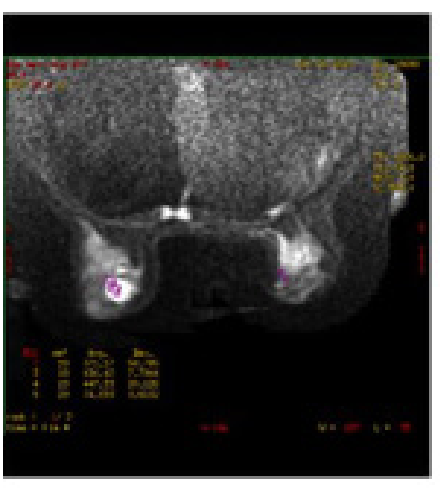

A

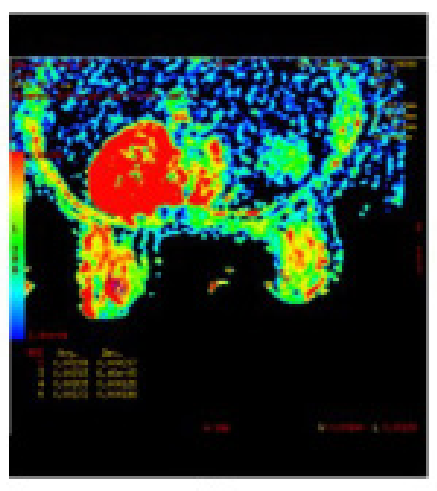

B

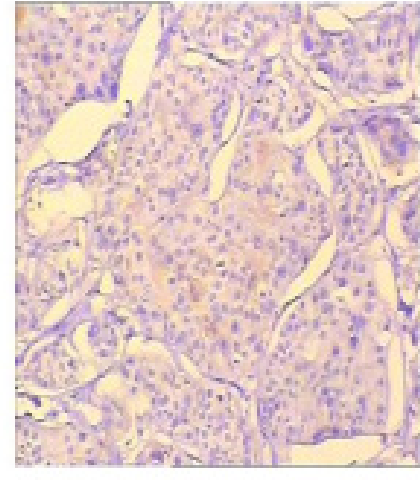

$\mathrm{C}$

Figure 2. Female patient, 47 years, left breast fibrocystic disease. A. Diffusion-weighted imaging shows an obvious high signal in the lesion of the left breast. B. Corresponding apparent diffusion coefficient (ADC) map: the average $\mathrm{ADC}$ value was $2.190 \times 10^{-3} \mathrm{~mm}^{2} / \mathrm{s}$, the region of interest area was approximately $19 \mathrm{~mm}^{2}$, and the relative apparent diffusion coefficient was 1.042 . C. Negative immunohistochemical staining for human epidermal growth factor receptor-2 (SP 400X).

Table 2. Correlation of ADC and rADC values with HER-2 expression in breast cancer.

\begin{tabular}{lccccc}
\hline HER-2 & Cases $(\mathrm{N}=64)^{*}$ & ADC & P & rADC & P \\
\hline$(-)$ & $45(70.3)$ & 1.109 & 0.923 & 0.8531 & 0.043 \\
$(+)$ & $2(3.1)$ & 1.127 & & 0.8204 & \\
$(++)$ & $4(6.3)$ & 1.098 & & 0.7483 & \\
$(+++)$ & $13(20.3)$ & 1.005 & & 0.6874 & \\
\hline
\end{tabular}

*Percentages are presented in parentheses. ADC $=$ apparent diffusion coefficient; rADC $=$ relative apparent diffusion coefficient; HER-2 = human epidermal growth factor receptor. 


\section{DISCUSSION}

The biological behavior of breast cancer can vary, and thus, the response of this malignancy to treatment can be variable. If the biological behavior of the tumor is understood and the therapeutic modality is selected on the basis of this behavior, then it is hoped that breast cancers with different biological behaviors can be treated with targeted remedies. The traditional prognostic factors include the metastatic status of axillary nodes, tumor size, and pathological grade. Traditional prognostic factors are simple and widely used, but the biological behavior of tumors can only be predicted at the macroscopic level. Early prediction is difficult, and its specificity is low. According to some studies, there is no correlation between the aforementioned traditional prognostic factors and ADC values (Kim et al., 2009). The most commonly used clinical prognostic factors include Ki-67, estrogen receptor status, progesterone receptor status, and HER-2 expression, and other factors under investigation include $\mathrm{p} 53$, epidermal growth factor receptor expression, and mitosis. The aforementioned molecular prognostic factors have high specificity, and some of the indices can be classified and quantified.

Water diffusion is impacted by many factors such as cell density, fluid viscosity, cell membrane permeability, active transport, and flow. Kim et al. (2007) and others found that the cells of benign breast cancer lesions are dense with limited water diffusion. These lesions show high signal activity on DWI and a low ADC value, which agrees with the result of this study. Recently, some breast cancer researchers suggested that the ADC is influenced by Brownian motion and the perfusion status of capillary networks (Kim et al., 2009).

HER-2 is the most common proto-oncogene in breast cancer. In total, $25-30 \%$ of patients with primary breast cancer exhibit HER-2 over expression. Trastuzumab has been adopted in clinical practice to treat HER-2-overexpressing breast cancer because this drug controlled the increase in the number of HER-2-overexpressing tumor cells in extracorporeal and animal testing. HER-2-positive cells are typified by cell proliferation, invasion, and transfer, and HER-2 expression is related to poor cellular differentiation and a large tumor volume (Berry et al., 2006). Alterations in water diffusion via changes in proliferation and tumor volume will change the ADC and rADC values correspondingly. Conversely, HER-2 overexpression increases the importance of VEGF in guiding angiogenesis. Makkat et al. (2008) and others reported that HER-2 positivity is associated with higher tumor blood flow. Therefore, change in tumor molecules will affect instillation, which manifests as an increase in ADC values. The ADC is influenced by both water molecule diffusion (decreases the ADC) and tumor instillation (increases the ADC). In this study, the former played a more important role.

rADC is the ratio of the ADC values of lesions and normal breast tissue. This parameter can eliminate individual differences in the ADC values (Xie et al., 2010). The rADC has greater specificity for diagnosing breast lesions than the ADC (Xie et al., 2010).

The ADC and $\mathrm{rADC}$ are influenced by both water molecule diffusion and the status of tissue perfusion.

It has been proven that breast cancer x-ray mammography calcification is closely related with HER-2 overexpression ( $\mathrm{P}=0.001)$ (Tan et al., 2009), implying that the imaging features of breast cancer are correlated with HER-2. Our study find that Her-2 is correlated with the $\mathrm{rADC}$ and there are no correlation between Her-2 and the ADC value. The reason for this finding may be that the rADC value partly eliminates the individual differences affecting the absolute ADC values. 
Some studies failed to identify a correlation between the ADC value and HER-2 expression. Possible explanations for this finding is that the $b$ value is different in different studies, resulting in varying ADC values (Wang and Duan, 2009), and choosing a lower b value may slightly increase the ADC (Hatakenaka et al., 2008). However, a higher b value may result in image distortion due to the long TE (Woodhams et al., 2005). In previous studies, a proper $\mathrm{b}$ value facilitated diagnosis, provided a quality picture, and more accurately clarified the tissue diffusion coefficient. One study suggested that the $b$ value always exceeds $400 \mathrm{~s} /$ $\mathrm{mm}^{2}$ (Le Bihan et al., 1988), but in our study, the b value was $800 \mathrm{~s} / \mathrm{mm}^{2}$. Gu et al. (2007) and others concluded that greater accuracy of the ADC is not necessary, arguing that meeting the requirement of diagnosis and differential diagnosis is sufficient. However, no prior study clarified the optimal value of $b$. In addition, the magnetic field may also influence DWI. In the study of 3.0T DWI (Lo et al., 2009), when the b value was unchanged, the ADC signalnoise ratio of 3.0T was higher than that of $1.5 \mathrm{~T}$. However, the limitation of that study was that the pathological pattern was too simple, potentially resulting in bias caused by histological changes (60 invasive ductal carcinomas, accounting for $93.75 \%$ of all lesions).

Overall, the rADC value is negatively correlated with HER-2 expression in patients with breast cancer. The ADC and rADC values are determined by water molecule diffusion and tissue perfusion.

\section{ACKNOWLEDGMENT}

Research supported by the project of Taishan Scholars Construction Engineering.

\section{REFERENCES}

Berry DA, Cirrincione C, Henderson IC, Citron ML, et al. (2006). Estrogen-receptor status and outcomes of modern chemotherapy for patients with node-positive breast cancer. JAMA 295: 1658-1667.

Cell Markers and Cytogenetics Committees, College of American Pathologists (2002). Clinical laboratory assays for HER-2/neu amplification and overexpression: quality assurance, standardization, and proficiency testing. Arch. Pathol. Lab. Med. 126: 803-808.

Ellis IO, Dowsett M, Bartlett J, Walker R, et al. (2000). Recommendations for HER2 testing in the UK. J. Clin. Pathol. 53: 890-892.

Gu YJ and Qiu LH (2007). Diffusion in the diagnosis of breast lesions. Zhong Guo Yi Xue Ji Suan Ji Cheng Xiang Za Zhi 13: 356-361.

Hatakenaka M, Soeda H, Yabuuchi H, Matsuo Y, et al. (2008). Apparent diffusion coefficients of breast tumors: clinical application. Magn. Reson. Med. Sci. 7: 23-29.

Kim CK, Park BK, Han JJ, Kang TW, et al. (2007). Diffusion-weighted imaging of the prostate at $3 \mathrm{~T}$ for differentiation of malignant and benign tissue in transition and peripheral zones: preliminary results. J. Comput. Assist. Tomogr. 31: 449-454.

Kim SH, Cha ES, Kim HS, Kang BJ, et al. (2009). Diffusion-weighted imaging of breast cancer: correlation of the apparent diffusion coefficient value with prognostic factors. J. Magn. Reson. Imaging 30: 615-620.

Le Bihan D, Breton E, Lallemand D, Aubin ML, et al. (1988). Separation of diffusion and perfusion in intravoxel incoherent motion MR imaging. Radiology 168: 497-505.

Lo GG, Ai V, Chan JK, Li KW, et al. (2009). Diffusion-weighted magnetic resonance imaging of breast lesions: first experiences at 3 T. J. Comput. Assist. Tomogr. 33: 63-69.

Makkat S, Luypaert R, Stadnik T, Bourgain C, et al. (2008). Deconvolution-based dynamic contrast-enhanced MR imaging of breast tumors: correlation of tumor blood flow with human epidermal growth factor receptor 2 status and clinicopathologic findings-preliminary results. Radiology 249: 471-482.

Tan XH, Xia SL, Ouyang YL and Luo HJ (2009). Molybdenum target X-ray features of breast cancer and ER, PR and HER-2 Correlation. Lin Chuang Fang She Xue Za Zhi 28: 1618-1622. 
Wang SY and Duan Q (2009). The value of diffusion weighted imaging in the differential diagnosis of benign and malignant breast lesions at 3.0 T MRI. Zhong Guo CT He MRI Za Zhi 7: 18-21.

Woodhams R, Matsunaga K, Iwabuchi K, Kan S, et al. (2005). Diffusion-weighted imaging of malignant breast tumors: the usefulness of apparent diffusion coefficient (ADC) value and ADC map for the detection of malignant breast tumors and evaluation of cancer extension. J. Comput. Assist. Tomogr. 29: 644-649.

Xie CM, Yin SH, Li H and Liu XW (2010). Diffusion of apparent diffusion coefficient and relative apparent diffusion coefficient value in diagnosis of malignant breast tumor table weighted imaging. Zhonghua Zhong Liu Za Zhi 32: 217-220. 